\title{
A First Order Method for Differential Equations of Neutral Type
}

\author{
By R. N. Castleton and L. J. Grimm*
}

\begin{abstract}
A first order method is presented for solution of the initial-value problem for a differential equation of neutral type with implicit delay in the critical case where the time-lag is zero and the method of stepwise integration does not apply. A convergence theorem is proved, and numerical examples are given.
\end{abstract}

1. Introduction. In this note, we present a first order method for the numerical solution of the initial-value problem (IVP) for a neutral-type functional-differential equation without previous history:

$$
\begin{aligned}
x^{\prime}(t) & =f\left(t, x(t), x(g(t, x(t))), x^{\prime}(g(t, x(t)))\right), \\
x(a) & =x_{0}, \quad x^{\prime}(a)=z_{0},
\end{aligned}
$$

where $z_{0}$ is a real root of the algebraic equation

$$
z=f\left(a, x_{0}, x_{0}, z\right) \text {. }
$$

Here, $x(t)$ is a scalar function to be determined on some finite interval $[a, b]$. We shall make the following assumptions regarding $f$ and $g$ :

(H1) $f$ and $g$ are continuous and satisfy uniform Lipschitz conditions of the form

$$
\begin{aligned}
\left|f\left(t, x_{1}, y_{1}, z_{1}\right)-f\left(t, x_{2}, y_{2}, z_{2}\right)\right| & \leqq L\left\{\left|x_{1}-x_{2}\right|+\left|y_{1}-y_{2}\right|\right\}+L_{z}\left|z_{1}-z_{2}\right|, \\
\left|g\left(t, x_{1}\right)-g\left(t, x_{2}\right)\right| & \leqq L_{o}\left|x_{1}-x_{2}\right|
\end{aligned}
$$

in their respective domains $E$ and $E^{\prime}$, where

$$
E=\left\{(t, x, y, z): a \leqq t \leqq b,\left|x-x_{0}\right| \leqq c,\left|y-x_{0}\right| \leqq c,|z| \leqq M\right\}
$$

and $E^{\prime}$ is the projection of $E$ in the $(t, x)$ space; $c, M, L, L_{o}, L_{z}$ are constants, with $L_{z}<1, M$ is such that $\sup _{(t, x, y, z) \in E}|f(t, x, y, z)|<M$, and $M(b-a)<c$.

(H2) $a \leqq g(t, x) \leqq t$ for $(t, x) \in E^{\prime}$.

Our hypotheses, together with additional smoothness and growth conditions on $f$ and $g$, ensure the local existence of a solution of the IVP (1)-(2). Furthermore, $x(t)$ is the only solution having a bounded derivative on [a, b]; see [2], [4]. Our result extends a method developed by Feldstein [3] for the equation of retarded type

Received August 7, 1970.

AMS (MOS) subject classifications (1970). Primary 34K99; Secondary 65L05.

Key words and phrases. Equations of neutral type, functional-differential equations, implicitdelay equations, numerical methods.

* Research of second author supported by National Science Foundation. 


$$
x^{\prime}(t)=f(t, x(t), x(g(t)))
$$

to the neutral-type equation with implicit delay (1). Other methods for implicitdelay equations are given in [1].

2. The Algorithm $\mathfrak{A}$. Let $y(t)=x(g(t, x(t))) ; z(t)=x^{\prime}(g(t, x(t)))$. Let $N$ be a positive integer, and let $h=(b-a) / N$. For each nonnegative integer $n \leqq N$, let $t_{n}=a+n h$. Let $[s]$ denote the integer part of $s$. Define the algorithm $\mathfrak{A}$ as follows:

$$
\begin{aligned}
f_{n} & =f\left(t_{n}, x_{n}, y_{n}, z_{n}\right), & g_{n} & =g\left(t_{n}, x_{n}\right), \\
q(n) & =\left[\left(g_{n}-a\right) / h\right], & r(n) & =\left(g_{n}-a\right) / h-q(n), \\
y_{0} & =x_{0}, & & \\
z_{n} & =f_{a(n)}, & & \\
x_{n+1} & =x_{n}+h f_{n} . & &
\end{aligned}
$$

Note that condition (H2) implies $q(n) \leqq n$, thus, the algorithm is well defined. For $n=0, g_{0}=a, q(0)=0$, and $r(0)=0$. Thus, $y_{0}=x_{0}$ and $z_{0}=f\left(a, x_{0}, x_{0}, z_{0}\right)$. Let $u_{0}$, an approximation of the root $z_{0}$, be chosen independently of $h$. It is of interest to note that such an approximation does not destroy the order $h$ convergence of the algorithm. It is of further interest that (6) may be simplified to $y_{n}=x_{a(n)}$. The error bound established in the convergence theorem for this "simplified" algorithm is larger but still of order $h$, as noted following the proof of convergence of the algorithm $\mathfrak{A}$. The second numerical example of Section 4 demonstrates both the algorithm $\mathfrak{A}$ and the simplified algorithm.

If $g_{n}=t_{n}$ for any $n, 1 \leqq n \leqq N$, then $q(n)=n, r(n)=0$, and (7) becomes $z_{n}=$ $f\left(t_{n}, x_{n}, y_{n}, z_{n}\right)$ which has exactly one root $z$ in the interval $[-M, M]$ under the conditions $(\mathrm{H} 1)-(\mathrm{H} 2)$ together with the smoothness and growth conditions mentioned in Section 1. We must in general include a procedure for finding this root, and this in turn will affect the error estimate. As before, such an estimate does not destroy the order $h$ convergence of the algorithm. For simplicity, we do not take this into account, since our aim is to show the convergence of the algorithm $\mathfrak{A}$.

Thus, we shall assume in the convergence proof that (7) will not reduce to $z_{n}=$ $f\left(t_{n}, x_{n}, y_{n}, z_{n}\right), n \geqq 1$.

\section{Convergence.}

THEOREM. Let $f$ and $g$ satisfy $(\mathrm{H} 1)-(\mathrm{H} 2)$ and suppose, in addition, that there exists a unique solution $x(t)$ of (1)-(2) with $\sup _{|a, b|}\left|x^{\prime \prime}(t)\right| \leqq B$. Then, for each $t_{n} \in$ $[a, b], 0<n \leqq N$,

$$
\left|x_{n}-x\left(t_{n}\right)\right| \leqq h\left\{L_{z}\left|z_{0}-u_{0}\right| e^{s(b-a)}+\frac{B}{2 s}\left(\frac{1}{1}+\frac{L_{z}}{L_{z}}\right)\left(e^{s(b-a)}-1\right)\right\}+O\left(h^{2}\right)
$$

where

$$
s=L\left(1+c_{0}\right)+L_{2} c_{1}
$$




$$
\begin{aligned}
& c_{0}=1+M L_{v}, \\
& c_{1}=\left(L\left(2+M L_{u}\right)+B L_{\vartheta}\right) /\left(1-L_{z}\right),
\end{aligned}
$$

$u_{0}$ is the approximation to $z_{0}$ mentioned above, and $x_{n}$ is given by algorithm $\mathfrak{A}$.

Proof. Let $e_{n}=\left|x_{n}-x\left(t_{n}\right)\right| ; e_{n}^{*}=\left|y_{n}-y\left(t_{n}\right)\right| ; e_{n}^{* *}=\left|z_{n}-z\left(t_{n}\right)\right|$. From (8) and Taylor's formula, we obtain

$$
e_{n+1} \leqq e_{n}+h\left(L\left(e_{n}+e_{n}^{*}\right)+L_{z} e_{n}^{* *}\right)+h^{2} B / 2 .
$$

Equation (5) implies that $g_{n}=t_{a(n)}+h r(n)$, and hence, in a similar manner, we have (after replacing $n$ by $(n+1)$ )

$$
\begin{aligned}
e_{n+1}^{*} \leqq & M L_{0} e_{n+1}+e_{q(n+1)} \\
& +h r(n+1)\left\{L\left(e_{u(n+1)}+e_{u(n+1)}^{*}+L_{z} e_{q(n+1)}^{* *}\right\}+h^{2} r^{2}(n+1) B / 2,\right. \\
e_{n+1}^{* *} \leqq & B L_{u} e_{n+1}+L\left(e_{q(n+1)}+e_{q(n+1)}^{*}\right)+L_{z} e_{u(n+1)}^{* *}+h r(n+1) B .
\end{aligned}
$$

We then have two cases to consider:

Case 1. $q(n+1)=n+1$ and $r(n+1)=0$. Under these conditions, (9) is unchanged:

$$
e_{n+1} \leqq e_{n}(1+h L)+e_{n}^{*} h L+e_{n}^{* *} h L_{z}+h^{2} B / 2
$$

(10) becomes

$$
e_{n+1}^{*} \leqq e_{n+1}\left(1+M L_{v}\right)=e_{n+1} c_{0}
$$

And (11) becomes

$$
e_{n+1}^{* *} \leqq\left(L+B L_{u}\right) e_{n+1}+L e_{n+1}^{*}+L_{z} e_{n+1}^{* *}
$$

or

$$
e_{n+1}^{* *} \leqq\left(\frac{L+B L_{u}+L\left(1+M L_{0}\right)}{1-L_{z}}\right) e_{n+1}=e_{n+1} c_{1}
$$

Define the partial ordering for vectors: $v_{1}=\left(v_{1}^{1}, \cdots, v_{1}^{k}\right) \leqq v_{2}=\left(v_{2}^{1}, \cdots, v_{2}^{k}\right)$ if $v_{1}^{i} \leqq v_{2}^{i}, i=1, \cdots, k$. Then, in vector form, (9a), (10a), and (11a) become

$$
\left[\begin{array}{l}
e_{n+1} \\
e_{n+1}^{*} \\
e_{n+1}^{* *}
\end{array}\right] \leqq\left[\begin{array}{ccc}
1+h L & h L & h L_{z} \\
(1+h L) c_{0} & h L c_{0} & h L_{z} c_{0} \\
(1+h L) c_{1} & h L c_{1} & h L_{2} c_{1}
\end{array}\right]\left[\begin{array}{c}
e_{n} \\
e_{n}^{*} \\
e_{n}^{* *}
\end{array}\right]+h B\left[\begin{array}{c}
h / 2 \\
h c_{0} / 2 \\
h c_{1} / 2
\end{array}\right]
$$

which is of the form $d_{n+1} \leqq A_{1} d_{n}+b_{1}$.

Case 2. $q(n+1) \leqq n$ and $0 \leqq r(n+1)<1$.

Let

$$
\delta_{n}=\max _{1 \leqq i \leqq n} e_{i}, \quad \delta_{n}^{*}=\max _{1 \leqq i \leqq n} e_{i}^{*}, \quad \delta_{n}^{* *}=\max _{1 \leqq i \leqq n} e_{i}^{* *} .
$$

Then, (9) becomes

$$
\delta_{n+1} \leqq \delta_{n}(1+h L)+\delta_{n}^{*} h L+\delta_{n}^{* *} h L_{z}+h^{2} B / 2 .
$$


And (10) becomes

$$
\delta_{n+1}^{*} \leqq M L_{o} \delta_{n+1}+\delta_{n}(1+h L)+h L \delta_{n}^{*}+h L_{z} \delta_{n}^{* *}+h^{2} B / 2 .
$$

Using (9b), we have

$$
\delta_{n+1}^{*} \leqq\left(\delta_{n}(1+h L)+\delta_{n}^{*} h L+\delta_{n}^{* *} h L_{z}+h^{2} B / 2\right)\left(1+M L_{o}\right)
$$

or

$$
\delta_{n+1}^{*} \leqq \delta_{n}(1+h L) c_{0}+\delta_{n}^{*} h L c_{0}+\delta_{n}^{* *} h L_{2} c_{0}+h^{2} c_{0} B / 2 .
$$

Finally, (11) becomes

$$
\delta_{n+1}^{* *} \leqq \delta_{n+1} B L_{o}+\delta_{n} L+\delta_{n}^{*} L+\delta_{n}^{* *} L_{z}+h B .
$$

Further, enlarging $\delta_{n}$ to $\delta_{n+1}$ and $\delta_{n}^{*}$ to $\delta_{n+1}^{*}$ on the right, and using $1-L_{z}>0$, we find

$$
\delta_{n+1}^{* *} \leqq \delta_{n+1}\left(\frac{L+B L_{0}}{1-L_{z}}\right)+\delta_{n+1}^{*} \frac{L}{1-L_{z}}+\frac{h B}{1-L_{z}} .
$$

Using (9b) and (10b), we have

$$
\delta_{n+1}^{* *} \leqq\left(\frac{L+B L_{o}+L c_{0}}{1-L_{z}}\right)\left(\delta_{n}(1+h L)+\delta_{n}^{*} h L+\delta_{n}^{* *} h L_{z}+\frac{h^{2} B}{2}\right)+\frac{h B}{1-L_{z}}
$$

or

(11b) $\delta_{n+1}^{* *} \leqq \delta_{n}(1+h L) c_{1}+\delta_{n}^{*} h L c_{1}+\delta_{n}^{* *} h L_{2} c_{1}+\frac{h B}{1-L_{z}}+\frac{h^{2} c_{1} B}{2}$.

Then, as a vector system, (9b), (10b), and (11b) become

$$
\left[\begin{array}{c}
\delta_{n+1} \\
\delta_{n+1}^{*} \\
\delta_{n+1}^{* *}
\end{array}\right] \leqq\left[\begin{array}{ccc}
1+h L & h L & h L_{z} \\
(1+h L) c_{0} & h L c_{0} & h L_{2} c_{0} \\
(1+h L) c_{1} & h L c_{1} & h L_{2} c_{1}
\end{array}\right]\left[\begin{array}{c}
\delta_{n} \\
\delta_{n}^{*} \\
\delta_{n}^{* *}
\end{array}\right]+h B\left[\begin{array}{c}
h / 2 \\
h c_{0} / 2 \\
h c_{1} / 2+1 /\left(1-L_{z}\right)
\end{array}\right]
$$

which is of the form $d_{n+1} \leqq A_{2} d_{n}+b_{2}$. Comparing this with the result obtained in Case 1 , we find that $A_{1}$ and $A_{2}$ are identical and that $b_{1} \leqq b_{2}$. Thus, any bound obtained here in Case 2 for $d_{n+1}$ will also bound $d_{n+1}$ in Case 1 .

To complete the proof, we shall use the following lemmas [3] which may be verified by induction:

Lemma 1. Suppose $A$ is a $k \times k$ real matrix and $b$ is a real $k$-vector. Let $\left\{d_{n}\right\}$ $(n=0,1, \cdots)$ satisfy $d_{n+1} \leqq A d_{n}+b$. Then

$$
d_{n+1} \leqq A^{n+1} d_{0}+\left(\sum_{i=0}^{n} A^{i}\right) b .
$$

Lemma 2. Let $p=\left(p_{1}, \cdots, p_{k}\right), q=\left(q_{1}, \cdots, q_{k}\right)$. Suppose the $k \times k$ matrix $A$ has the form $A=p^{T} q$. Then

$$
A^{n}=\left(\sum_{i=1}^{k} p_{i} q_{i}\right)^{n-1} A .
$$


By Lemma 1,

$$
d_{n+1} \leqq A_{2}^{n+1} d_{0}+\left(\sum_{i=1}^{n} A_{2}^{i}\right) b_{2}
$$

where

$$
d_{01}=\left[\begin{array}{c}
e_{01} \\
e_{0}^{*} \\
e_{01}^{* *}
\end{array}\right]=\left[\begin{array}{c}
0 \\
0 \\
\left|z_{0}-u_{0}\right|
\end{array}\right] .
$$

Then, because

$$
A_{2}=\left[\begin{array}{c}
1 \\
c_{0} \\
c_{1}
\end{array}\right]\left(1+h L, h L, h L_{2}\right),
$$

we can make use of Lemma 2 to obtain

$$
A_{2}^{i}=\left(1+h L+h L c_{1}+h L_{2} c_{1}\right)^{i-1} A_{2}=(1+h s)^{i-1} A_{2} .
$$

Two results follow from this: $A_{2}^{n+1}=(1+h s)^{n} A_{2} \leqq e^{s(b-a)} A_{2}$, and

$\sum_{i=1}^{n} A_{2}^{i}=A_{2} \sum_{i=1}^{n}(1+h s)^{i-1}=\frac{\left((1+h s)^{n}-1\right)}{h s} A_{2} \leqq \frac{1}{h s}(\exp (s(b-a))-1) A_{2}$.

Finally,

$$
\begin{aligned}
& d_{n+1} \leqq A_{2}^{n+1} d_{0}+\left(\sum_{i=0}^{n} A_{2}^{i}\right) b_{2} \\
& \leqq h\left\{z_{11}-u_{01} \mid L_{2} e^{e(1)-n)}\left[\begin{array}{l}
1 \\
c_{0} \\
c_{1}
\end{array}\right]\right.
\end{aligned}
$$

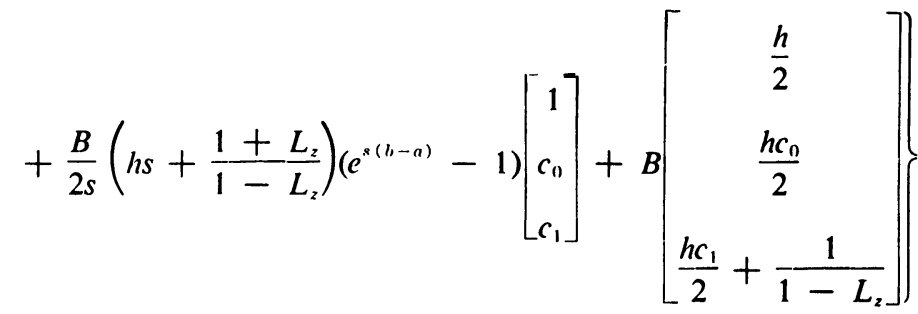

which gives

$$
c_{n+1} \leqq \delta_{n+1} \leqq h\left\{\left|z_{n}-u_{0}\right| L_{z} e^{*(h-a)}+\frac{B}{2 s}\left(h s+\frac{1+L_{z}}{1-L_{z}}\right)\left(e^{*(b-a)}-1\right)+\frac{h B}{2}\right\}
$$

and the theorem follows. 
For the simplified algorithm, where (6) is replaced by $y_{n}=x_{a(n)}$ the following bound is possible:

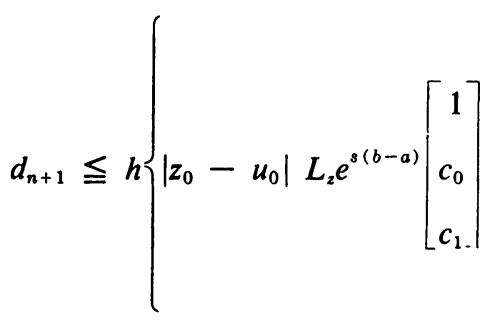

$$
\begin{aligned}
+\left(\frac{B}{2 s}\left(h s+\frac{1+L_{z}}{1-L_{z}}\right)+\frac{1}{s}\left(\frac{M L}{1-L_{z}}\right)\right)\left(e^{3(b-a)}-1\right)\left[\begin{array}{c}
1 \\
c_{0} \\
c_{1 .}
\end{array}\right] \\
\left.+B\left[\begin{array}{c}
\frac{h}{2} \\
\frac{h c_{0}}{2} \\
\frac{h c_{1}}{2}+\frac{1}{1-L_{z}}
\end{array}\right]+\left[\begin{array}{c}
0 \\
M \\
\frac{M L}{1-L_{z}}
\end{array}\right]\right\},
\end{aligned}
$$

and hence

$$
\begin{aligned}
e_{n+1} \leqq & \left\{\left|z_{0}-u_{0}\right| L_{2} e^{s(b-a)}\right. \\
& \left.+\left(\frac{B}{2 s}\left(h s+\frac{1+L_{z}}{1-L_{z}}\right)+\frac{1}{s}\left(\frac{M L}{1-L_{z}}\right)\right)\left(e^{s(b-a)}-1\right)+\frac{h B}{2}\right\} .
\end{aligned}
$$

TABLE I. $x_{n}(h)$ denotes the value of $x_{n}$ for step size $h$.

\begin{tabular}{llllll}
\hline \multicolumn{1}{c}{$t_{n}$} & $x\left(t_{n}\right)$ & $x_{n}\left(2^{-4}\right)$ & $x_{n}\left(2^{-6}\right)$ & $x_{n}\left(2^{-8}\right)$ & $x_{n}\left(2^{-10}\right)$ \\
\hline 0 & 0 & 0 & 0 & 0 & 0 \\
.0625 & .0039 & 0 & .0029 & .0034 & .0039 \\
.1250 & .0158 & .0078 & .0138 & .0153 & .0157 \\
.1875 & .0360 & .0238 & .0329 & .0352 & .0358 \\
.2500 & .0653 & .0484 & .0610 & .0642 & .0650 \\
.3125 & .1048 & .0825 & .0990 & .1032 & .1044 \\
.3750 & .1562 & .1275 & .1485 & .1541 & .1556 \\
.4375 & .2224 & .1853 & .2119 & .2196 & .2217 \\
.5000 & .3078 & .2593 & .2942 & .3043 & .3069 \\
.5625 & .4206 & .3547 & .4026 & .4159 & .4194 \\
.6250 & .5771 & .4856 & .5518 & .5705 & .5754 \\
.6875 & .8185 & .6707 & .7778 & .8080 & .8159 \\
.7500 & 1.3244 & .9860 & 1.2205 & 1.2968 & 1.3174 \\
\hline
\end{tabular}


TABLE II. $x_{n}^{(1)}(h)$ denotes the value of $x_{n}$ for step size $h$ by algorithm $\mathfrak{A} ; x_{n}^{(2)}(h)$ denotes the value of $x_{n}$ for step size $h$ by the simplified algorithm.

\begin{tabular}{cccccc}
\hline$t_{n}$ & $x\left(t_{n}\right)$ & $x_{n}^{(1)}\left(2^{-2}\right)$ & $x_{n}^{(2)}\left(2^{-2}\right)$ & $x_{n}^{(1)}\left(2^{-4}\right)$ & $x_{n}^{(2)}\left(2^{-4}\right)$ \\
\hline .25 & .2474 & .2500 & .2500 & .2483 & .2478 \\
.50 & .4794 & .4930 & .4892 & .4838 & .4759 \\
.75 & .6816 & .7180 & .6866 & .6942 & .6739 \\
1.00 & .8414 & .9228 & .8569 & .8697 & .8273 \\
$t_{n}$ & $x\left(t_{n}\right)$ & $x_{n}^{(1)}\left(2^{-8}\right)$ & $x_{n}^{(2)}\left(2^{-8}\right)$ & $x_{n}^{(1)}\left(2^{-12}\right)$ & $x_{n}^{(2)}\left(2^{-12}\right)$ \\
.25 & .2474 & .2475 & .2471 & .2474 & .2474 \\
.50 & .4794 & .4797 & .4787 & .4794 & .4794 \\
.75 & .6816 & .6825 & .6802 & .6817 & .6815 \\
1.00 & .8414 & .8435 & .8390 & .8416 & .8413 \\
\hline
\end{tabular}

4. Examples. (a) We solve the IVP

$$
x^{\prime}(t)=\frac{-4 t x^{2}(t)}{4+\log ^{2} \cos t}+\tan 2 t+\frac{1}{2} \tan ^{-1} z
$$

$\left(z_{0}=0, x_{0}=0, z=x^{\prime}(g(t, x(t))) \equiv x^{\prime}\left(t x^{2}(t) /\left(1+x^{2}(t)\right)\right)\right)$ on the interval $[0, .75]$. The existence and uniqueness of the solution is guaranteed by the results of [2] mentioned earlier. The only solution is $x(t)=-\frac{1}{2} \log \cos 2 t$.

The results of the computation by algorithm $\mathfrak{A}$ are given in Table $\mathrm{I}$.

(b) Consider the IVP

$$
x^{\prime}(t)=\cos t(1+y)+x z-\sin \left(t\left(1+\sin ^{2} t\right)\right),
$$

with $y=x\left(t x^{2}(t)\right), z=x^{\prime}\left(t x^{2}(t)\right), z_{0}=1, x_{0}=0$, on the interval [0,1]. As in example (a), existence and uniqueness of the solution are guaranteed by the results of [2]. Here, the solution is $x(t)=\sin t$.

The results of the computation by the algorithm $\mathfrak{A}$ and by the simplified algorithm are given in Table II.

Computation Division

Lawrence Livermore Laboratory

Livermore, California 94551

Department of Mathematics

University of Missouri, Rolla

Rolla, Missouri 65401

1. R. N. Castleton, Some Numerical Methods for Differential Equations with Deviating Arguments, M.S. Thesis, University of Utah, 1970.

2. R. N. CASTLETON \& L. J. GRIMM, "Local existence and uniqueness for neutral functional equations." (In preparation.)

3. M. A. FeldSTEIN, Discretization Methods for Retarded Ordinary Differential Equations, Ph.D. Thesis, University of California, Los Angeles, Calif., 1964.

4. L. J. GRIMM, "Existence and continuous dependence for a class of nonlinear neutraldifferential equations," Proc. Amer. Math. Soc., v. 29, 1971, 467-473. MR 44 \#4324. 